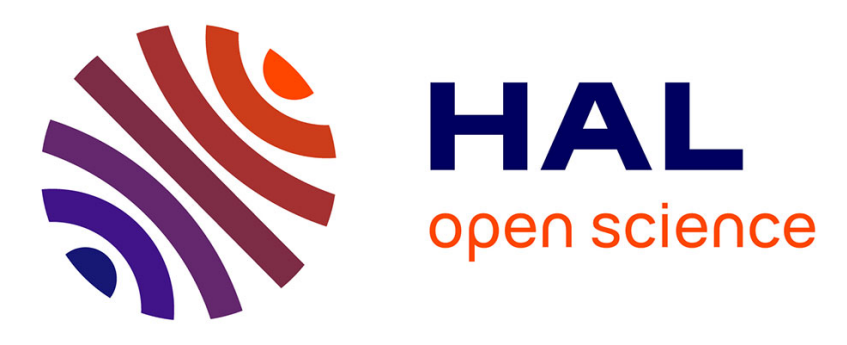

\title{
Analysis of lymph node tumor features in PET/CT for segmentation
}

\author{
Diana Lucia Farfan Cabrera, Eloïse Grossiord, Nicolas Gogin, Dimitri \\ Papathanassiou, Nicolas Passat
}

\section{To cite this version:}

Diana Lucia Farfan Cabrera, Eloïse Grossiord, Nicolas Gogin, Dimitri Papathanassiou, Nicolas Passat. Analysis of lymph node tumor features in PET/CT for segmentation. International Symposium on Biomedical Imaging (ISBI), 2021, Nice, France. pp.588-592, 10.1109/ISBI48211.2021.9433791 . hal03104417

\section{HAL Id: hal-03104417 https://hal.science/hal-03104417}

Submitted on 12 Apr 2021

HAL is a multi-disciplinary open access archive for the deposit and dissemination of scientific research documents, whether they are published or not. The documents may come from teaching and research institutions in France or abroad, or from public or private research centers.
L'archive ouverte pluridisciplinaire HAL, est destinée au dépôt et à la diffusion de documents scientifiques de niveau recherche, publiés ou non, émanant des établissements d'enseignement et de recherche français ou étrangers, des laboratoires publics ou privés. 


\title{
ANALYSIS OF LYMPH NODE TUMOR FEATURES IN PET/CT FOR SEGMENTATION
}

\author{
D.L. Farfan Cabrera ${ }^{1,2}$, É. Grossiord $^{3}$, N. Gogin ${ }^{2}$, D. Papathanassiou ${ }^{1,4}$, N. Passat ${ }^{1}$ \\ ${ }^{1}$ Université de Reims Champagne Ardenne, CReSTIC EA 3804, 51097 Reims, France \\ ${ }^{2}$ General Electric Healthcare, Buc, France \\ ${ }^{3}$ IMT, UMR CNRS 5219; 3IA ANITI; Université de Toulouse; F-31062 Toulouse Cedex 9, France \\ ${ }^{4}$ Institut Jean-Godinot, Reims, France
}

\begin{abstract}
In the context of breast cancer, the detection and segmentation of cancerous lymph nodes in PET/CT imaging is of crucial importance, in particular for staging issues. In order to guide such image analysis procedures, some dedicated descriptors can be considered, especially region-based features. In this article, we focus on the issue of choosing which features should be embedded for lymph node tumor segmentation from PET/CT. This study is divided into two steps. We first investigate the relevance of various features by considering a Random Forest framework. In a second time, we validate the expected relevance of the best scored features by involving them in a U-Net segmentation architecture. We handle the region-based definition of these features thanks to a hierarchical modeling of the PET images. This analysis emphasizes a set of features that can significantly improve / guide the segmentation of lymph nodes in PET/CT.
\end{abstract}

Index Terms - PET/CT, breast cancer, lymph nodes, tumor segmentation, region-based features, feature analysis, Random Forest, U-Net, component-tree

\section{INTRODUCTION}

Positron Emission Tomography (PET), generally coupled with X-ray Computed Tomography (CT), is widely used for medical imaging purpose. In particular, FDG $\left({ }^{18} \mathrm{~F}\right.$-Fluorodesoxyglucose) PET constitute a relevant source of information for diagnosis and patient follow-up in clinical applications related to cancer. As a consequence, PET/CT image analysis is an increasingly considered research topic. The issue of image analysis (detection, segmentation) from PET/CT images is considered for more than fifteen years [1], and the first automatic methods were pioneered ten years ago [2, 3].

Within the potential applications of computer-aided diagnosis based on PET/CT, the case of breast cancer $[4,5]$ is of paramount importance. Indeed, it is one of the most common diseases and one of the principal causes of death in women. This type of cancer develops from breast tissue, and lymph nodes (LN) near these regions are among the first structures to be affected. LN are then usually involved in protocols dedicated to the staging of breast cancer. This justifies the relevance of segmenting LN from PET/CT images, which allows to assess both their morphological and functional (possibly pathological) properties.

A limited literature concerning detection / segmentation of LN tumors in PET/CT is available. One can cite [6] that performs LN cluster segmentation in the thoracoabdominal area in 2D CT slices using firstly holistically-nested neural networks and then enhancing segmentation with optimization techniques. In [7] a method is proposed for axillary LN segmentation in CT using a classification of solid vs. non-solid LN depending on their tissue homogeneity.
In the context of medical imaging, Deep Learning (DL) has emerged as a promising paradigm. Convolutional Neural Networks (CNNs) initially developed in the fields of computer vision $[8,9]$ have led to impressive progress in medical image analysis [10], with dedicated architectures such as U-net [11] which is now a goldstandard. In the context of PET/CT analysis, it was shown in [12] that CNNs, already used for PET/CT co-segmentation [13], were a potentially relevant paradigm. In particular, DL outperforms other machine learning approaches for the task of LN segmentation [14].

Following that way, we recently proposed an LN segmentation method based on a U-Net architecture [15]. It was designed to take advantage from PET and CT information, but also from additional information, under the form of feature maps precomputed from the PET/CT images. Beyond the relevance of using DL-based methods, our working hypothesis is that high-level region-based features [16] computed from PET/CT images may be used for modeling knowledge useful for guiding the segmentation process. (Indeed, such high-level knowledge may not be directly infered by CNNs.)

Region-based features were previously investigated in the field of image analysis via hierarchical models. In particular, for functional imaging, a hierarchical model, the so-called component-tree (C-Tree) [17] was successfully involved over the last years. It allows to emphasize the regions of locally extremal values and is then well-suited for analyzing images where the strength of the signal is directly correlated to its relevance. C-Trees were considered for PET image segmentation via optimal cut determination [18, 19], attribute filtering [20, 21, 22] or machine learning approaches [23, 24].

Following the hypothesis that region-based features computed from PET/CT constitute a precious source of information for LN segmentation, our purpose in this article is (1) to describe a framework that allows to assess the relevance of a set of features, so that the best ones may be involved for segmentation guidance, and (2) to show in which extent these chosen best features may be embedded in a CNN segmentation framework for improving its performances.

With respect to (1) (Sec. 2), we build upon a method initially proposed in [24], that carries out classification in FDG PET/CT from region-based features. This first machine learning step relies on a Random Forest (RF) [25] approach on the PET/CT attributes computed from regions modeled in the C-Trees of PET images. In [24], this paradigm was used for segmentation purpose. By contrast, in our study, we take advantage of the ability of RFs to evaluate the relative impact of each feature on the quality of the classification, thus enabling to discriminate the potentially good features.

With respect to (2) (Sec. 3), we enrich a U-Net segmentation architecture initially described in [15], by embedding these potentially good features as additional inputs. An accurate analysis of the results (Sec. 4) then allows us to confirm / recuse the relevance of 
these features identified in (1).

\section{REGION-BASED FEATURE ANALYSIS}

In this section, we describe a framework for investigating the relevance of various region-based features for PET/CT image analysis.

\subsection{Component-trees for hierarchical image modeling}

In order to evaluate the relevance of region-based features, it is mandatory to subdivide the considered images into regions with appropriate homogeneity properties, so that the computed feature values make sense. For various kinds of images, such subdivision may be performed by superpixel approaches. However, in the more specific case of medical images, and a fortiori for functional images, the decomposition of the data by hierarchical models has demonstrated its relevance $[26,18]$.

In particular, we consider a decomposition of the PET images by the means of C-Trees [17]. A C-Tree is a rooted tree where each node corresponds to a connected component of a binary level-set of the image. These nodes / connected components are organized with respect to the inclusion relation $\subseteq$ on sets. When considering the $\leq$ relation on grey-level values of the image, the root of the C-Tree is the whole image support at the lowest value (here, 0 ). At the other side of the C-Tree, i.e. at the extremities of its branches, the leaves correspond to the flat zones of locally maximal values. In our case, these distal parts are areas of high uptake of the radioactive tracer, that correspond to high metabolism, induced e.g. by tumours.

The C-Tree can be used as an image processing tool, mainly for segmentation or anti-extensive filtering [27]. Since it is an image model, it can also be used to store, for each node, some feature values (also called attributes [16]) describing the properties of these nodes. This is the way we use C-Trees hereafter.

Practically, let $I: \Omega \rightarrow \mathbb{V}$ be a PET image defined on a 3D volume $\Omega \subset \mathbb{Z}^{3}$ and taking its values in a subset $\llbracket 0, V \rrbracket \subset \mathbb{Z}$. For each value $v \in \mathbb{V}$, let $I_{v} \subseteq \Omega$ be the binary level-set image corresponding to the thresholding of $I$ at value $v$, i.e. $I_{v}=\{x \in \Omega \mid v \leq I(x)\}$. Let $\mathcal{C}\left[I_{v}\right]$ be the set of all the connected components of $I_{v}$. Finally, let $\Theta=\bigcup_{v \in \mathbb{V}} \mathcal{C}\left[I_{v}\right]$ be the set of all these connected components for all the grey-level values. This set $\Theta$ corresponds to the set of the nodes of the $\mathrm{C}$-Tree, namely the regions that will be considered for our feature analysis. The C-Tree is the tree structure $(\Theta, \triangleleft)$ that corresponds to the Hasse diagram (i.e. the reflexive-transitive reduction) of the partially ordered set $(\Theta, \subseteq)$. Note that the lossless property of the $\mathrm{C}$-Tree wrt to the image $I$ is expressed by the following formula:

$$
I=\bigvee_{x \in \Omega} 1_{\{x\}} \max \left\{v \mid x \in N \in \Theta, N \subset I_{v}\right\}
$$

where $\bigvee$ is the pointwise supremum on functions and $1_{X}$ is the indicator function for the set $X$. (This formula will be involved in the feature map construction process of Sec. 3.2.)

\subsection{Feature computation}

Let us now suppose that the C-Tree has been built for the PET image $I$ (this can be done in quasi-linear time [28]). We now focus on the nodes $\Theta$ of the $\mathrm{C}$-Tree, that correspond to the regions of the image $I$ for which we want to compute the region-based features to be evaluated. By contrast with a usual partitioning, the regions of $\Theta$ given by a C-Tree form a cover of the image. In particular, some of these regions overlap (more precisely, some of them are included in others). This makes the region-based feature analysis richer.
For each region $N$ (i.e. for each node of $\Theta$ ) defined from the image, we compute several features. In this study, we chose to investigate 33 features that provide information on: (1) the geometry of $N$; (2) the statistics on the intensity distribution within $N$; and (3) the statistics on the spatial distribution of the intensity in $N$. Regarding the features of families $(2,3)$, we often studied PET and CT signal, by considering that both carry useful information. This arbitrary, yet large, set of features was chosen by assuming that the induced information cannot be easily inferred by CNNs (compared e.g. with spatial or contextual information). In other words, we chose the features the most likely to enrich a $\mathrm{CNN}$ architecture. lowing:

More precisely, the computed region-based features are the fol-

(1) shape features (11): eccentricity, elongation, sphericity, noncompacity, flatness, aspect ratio, bounding box height, width and depth, volume, inclusion in the convex hull of the lungs;

(2) spectral features (8): mean value and standard deviation (PET, CT), kurtosis (PET), skewness (PET), mean gradient border (PET), Shannon entropy (PET);

(3) texture features (mainly derived from the co-occurrence matrix [29]) (14): sparseness (PET), inverse difference moment (PET, CT), inertia (PET, CT), entropy (PET, CT), cluster prominence (PET, CT), energy (PET, CT), cluster shade (PET, CT) and relative integral volume [15] (PET).

From this analysis, for each region $N$, a feature vector $F_{N} \in \mathbb{R}^{33}$ was computed. This process was then iterated for all the regions $N$ of the C-Trees corresponding to all the couples of PET/CT images involved in the study (here 52 patients).

\subsection{Relevance study based on Random Forests}

Considering all the images involved in our study, and all the induced nodes / regions, this leads us to a set of 28143 feature vectors that lie in the feature space $\mathbb{R}^{33}$. Some of the regions correspond to tumor areas, whereas the others do not (and correspond e.g. to normal metabolic activity or "background" in PET). To identify the nodes belonging to each of these two classes, we rely on pre-defined clinical expert segmentations of tumor regions. In particular, we have 1959 tumor vectors vs. 26184 non-tumor vectors.

Our purpose is now to understand how these two families of vectors could be discriminated within the feature space $\mathbb{R}^{33}$. More precisely, we aim to determine which of these 33 features are the more likely to allow such discrimination. To this end, we rely on a RF classification [25]. Indeed, beyond its ability to learn how to classify data based on various features, RFs also allow to quantify the discriminative power of these features.

The RF framework consists of an ensemble of decision trees trained in parallel, with each tree recursively partitioning the input dataset, based on a splitting at each node in the tree according to the value of one selected feature, until reaching purity in the leaves (stopping criterion used in this work). More specifically, the different individual trees in the RF are trained on various random subsets of the original training dataset (bootstrapping) and consider random subsets of features to compute each node split (feature bagging), in order to obtain unique decorrelated trees and to reduce the overall variance of the RF. The RF finally predicts the class (tumor vs. nontumor) of each vector by carrying out a majority vote among the individual predictions of all the decision trees.

In our experiments, the RF hyper-parameters were optimized using a grid-search algorithm. Hence, our RF consists of 5000 decision trees and the optimal feature at each tree node is determined 
according to the information gain (i.e. minimizing the Shannon entropy). The SMOTE technique [30] was used in order to address the imbalanced data problem.

A 52-fold cross validation was performed by leave-one-patientout to identify the most discriminative features, i.e. the most robust and stable / consistent towards patients variability. Each of these trainings produced a scoring of feature importance; these scorings were averaged, leading to the final results in Fig. 1.

\section{FEATURE-BASED U-NET IMPROVEMENT}

We now embed the features that provide the best performances in the $\mathrm{RF}$ framework into a $\mathrm{CNN}$ architecture. Doing so, we aim to validate the hypothesis of their relevance and to improve the behaviour of the CNN-based segmentation via input enrichment.

\subsection{Features Description}

The 4 features identified as the best by the RF analysis (Fig. 1) are the mean gradient border $(M G B)$, the inertia (Ine), the inverse difference moment $(I D M)$ and the cluster prominence $(C P)$ :

$$
\begin{aligned}
M G B & =\frac{1}{|B|} \sum_{x \in B}|\nabla I|(x) \\
\text { Ine } & =\sum_{i} \sum_{j}(i-j)^{2} p_{i, j} \\
I D M & =\sum_{i} \sum_{j} \frac{p_{i, j}}{1-(i-j)^{2}} \\
C P & =\sum_{i} \sum_{j}\left(i+j-\mu_{x}-\mu_{y}\right)^{4} p_{i, j}
\end{aligned}
$$

where $I$ is the PET image,C $B$ the set of pixels on the border of the region, $i$ and $j$ are the coordinates of the co-occurence matrix of $I$ in the region, $p_{i, j}$ is the value of the matrix at $(i, j), \mu_{x}=$ $\sum_{i} i \sum_{j} p_{i, j}$ and $\mu_{y}=\sum_{j} j \sum_{i} p_{i, j}$.

These 4 features (that represent $35 \%$ of the overall discriminative power of the RF, vs. $65 \%$ for the other 29 features) are obtained from the PET image, and 3 of them are texture features derived from the co-occurence matrix analysis (only MGB is a spectral feature, computed however on a specific part of the regions).

\subsection{Feature Map Computation}

We aim to embed these 4 features $F$ as inputs of a $\mathrm{CNN}$ architecture, in addition to the two input PET and CT images of a given patient. To this end, these features $F$ have to be modeled the same way as these images, i.e. as maps $M_{F}: \Omega \rightarrow \mathbb{R}$.

The computation of these features was carried out region-wise, with regions forming a cover of $\Omega$. Then, a point $x \in \Omega$ may belong to several regions. More precisely, $x$ belongs to a node of the C-Tree at level set $I(x)$ and to all the other nodes of the branch of the tree down to the root. As a consequence, for a given feature, many values are associated to a same point.

This requires to find a way of "flattening" these information in order to build feature maps that associate exactly one value to each point. In other words, we aim to define a mapping:

$$
\operatorname{Flat}_{F}(x)=\operatorname{op}\{F(N) \mid x \in N \in \Theta\} \in \mathbb{R}
$$

associated to the feature $F$. Based on the analysis of the semantics of the features of Eqs. (2-5), we define op as $\max$ for $M G B$,

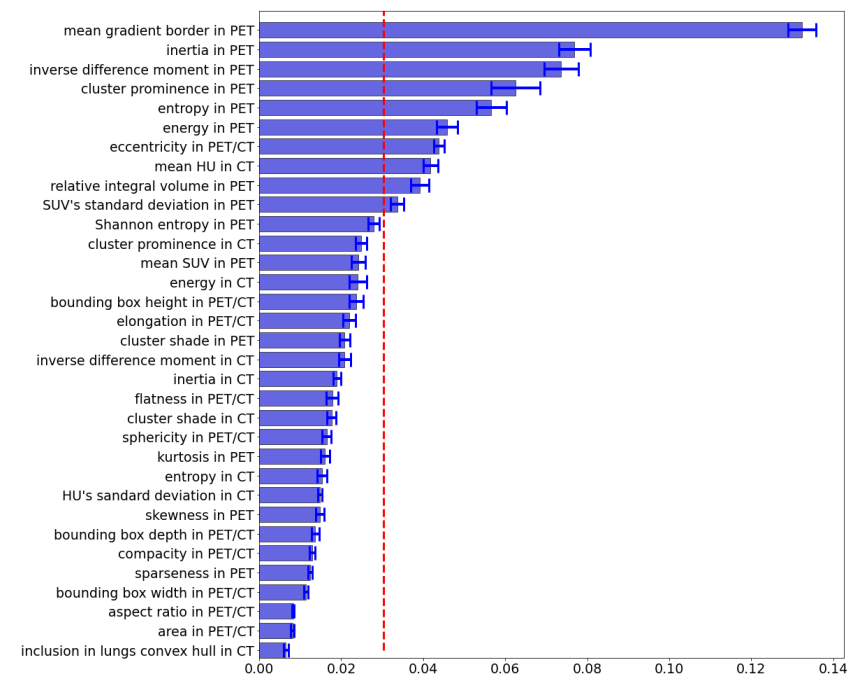

Fig. 1. Relative importance of the 33 explored features (Sec. 2.2) based on the RF study (Sec. 2.3). In blue: mean value and standard deviation of the relative importance of the features $\left(\sum=1\right)$; red line: mean relative importance value over the 33 features.
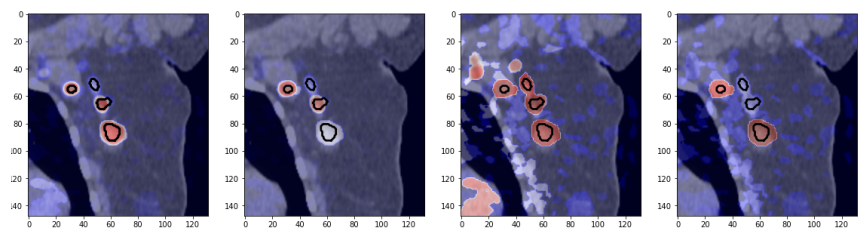

Fig. 2. Feature maps superimposed on a PET/CT image and viewed as heat-maps (blue $\rightarrow$ white $\rightarrow$ red, from low to high tumor probability). From left to right: $M G B$, Ine, $I D M$ and $C P$. Tumor $\mathrm{LN}$ ground-truth are depicted by black contours.

Ine, $C P$ and as min for $I D M$. (These choices were confirmed by experimental studies on the ability of the features to individually discriminate tumor from non-tumor regions.) Following the image reconstruction paradigm of Eq. (1), the definition of the feature maps is then:

$$
M_{F}=\bigvee_{x \in \Omega} 1_{\{x\}} \operatorname{Flat}_{F}(x)=\bigvee_{x \in \Omega} 1_{\{x\}} \mathrm{op}\{F(N) \mid x \in N \in \Theta\}
$$

Fig. 2 exemplifies the obtained feature maps corresponding to the features of Eqs. (2-5).

\subsection{Feature insertion into a 3D-UNet CNN}

Finally, these feature maps are inserted into a CNN architecture as supplementary channels, combined with the PET and CT images. The CNN (fully described in [15]) is based on a U-Net architecture with two encoders (one for the PET, the other for the CT) and one decoder for image reconstruction that carries out PET image reconstruction with the aid of skip connections deriving from the PET and CT encoders. Each encoder processes in parallel the information from PET and from CT respectively. The final output produces a heat-map with the probability of tumor location that can be thresholded for segmentation purpose. 
Table 1. Segmentation results without and with region-based feature enrichment (see Sec. 4). Sensitivity (Sen), Positive Predictive Value (PPV) and Dice scores are computed pixel-wise and region-wise (mean values and standard deviation).

\begin{tabular}{lrrrrrr}
\hline & & Pixel-wise & & & \multicolumn{2}{c}{ Region-wise } \\
Inputs & Sen & PPV & Dice & Sen & PPV & Dice \\
\hline PET/CT (baseline) & $0.887 \pm 0.046$ & $0.844 \pm 0.103$ & $0.865 \pm 0.080$ & $0.926 \pm 0.062$ & $0.850 \pm 0.189$ & $0.872 \pm 0.131$ \\
\hline PET/CT $+M G B$ & $0.856 \pm 0.055$ & $0.862 \pm 0.142$ & $0.850 \pm 0.016$ & $0.905 \pm 0.075$ & $0.838 \pm 0.194$ & $0.870 \pm 0.143$ \\
PET/CT + Ine & $0.879 \pm 0.041$ & $\mathbf{0 . 9 2 0} \pm 0.118$ & $0.890 \pm 0.019$ & $\mathbf{0 . 9 5 2} \pm 0.026$ & $\mathbf{0 . 8 8 2} \pm 0.116$ & $\mathbf{0 . 9 1 6} \pm 0.106$ \\
PET/CT + IDM & $0.862 \pm 0.121$ & $0.848 \pm 0.161$ & $0.855 \pm 0.066$ & $0.937 \pm 0.076$ & $0.868 \pm 0.120$ & $0.901 \pm 0.019$ \\
PET/CT + CP & $0.870 \pm 0.148$ & $0.833 \pm 0.121$ & $0.851 \pm 0.112$ & $0.937 \pm 0.013$ & $0.808 \pm 0.132$ & $0.868 \pm 0.145$ \\
\hline PET/CT + 4 & $\mathbf{0 . 9 1 0} \pm 0.018$ & $0.885 \pm 0.031$ & $\mathbf{0 . 8 9 7} \pm 0.024$ & $\mathbf{0 . 9 5 2} \pm 0.017$ & $0.870 \pm 0.026$ & $0.909 \pm 0.033$ \\
PET/CT + 5 [15] & $0.896 \pm 0.014$ & $0.872 \pm 0.012$ & $0.867 \pm 0.031$ & $0.933 \pm 0.015$ & $0.851 \pm 0.142$ & $0.894 \pm 0.133$ \\
\hline
\end{tabular}

\section{RESULTS AND DISCUSSION}

In this section, we provide results of the $\mathrm{CNN}$ with various kinds of inputs (data and feature maps):

- 2 inputs: PET and CT (i.e. without features): our baseline;

- 3 inputs: PET, CT plus one of the 4 features ( $M G B$, Ine, $I D M$ or $C P$ ) for assessing their individual impact;

- 6 inputs: PET, CT plus the 4 features $(M G B$, Ine, IDM and $C P$ ) for assessing their collective impact;

- 7 inputs: PET, CT plus 5 features empirically chosen in previous experiments [15], namely $M G B$, mean value (CT), standard deviation (CT), relative integral volume, inclusion in the convex hull of the lungs (that are ranked at the 1st, 8th, 25th, 9th and 33th positions, respectively in our current analysis).

The considered quality metrics are Sensitivity (Sen), Positive Predictive Value (PPV) and Dice score, computed point-wise (i.e. for segmentation) and region-wise (i.e. for detection), see Tab. 1. Segmentation results are also illustrated in Fig. 3.

From these results, one can first observe that the addition of feature maps as inputs generally has a positive effect on the results. However, only one additional feature map is often not enough. Indeed, except for Ine that improves the results vs. the baseline when added standalone, the other features marginally improve or slightly degrade the results in that case. By contrast, using several feature maps leads to improved (or in the worst cases to unchanged) results compared to the baseline. In particular, it leads to better scores in terms of true positives and false negatives compared to the case of standalone features, leading to a better sensitivity, which is important in a clinical context. Using various features also reduces the standard deviation of the scores, which denotes a more stable behaviour.

In the context of using several features, the last two lines of Tab. 1 seem to confirm that an accurate selection of these features (e.g. as proposed by the RF analysis) generally leads to better results compared to empirical choices. This emphasizes the relevance of the framework proposed in Secs. 2-3. However, we experimentally observed that involving too many features does not necessarily improve the $\mathrm{CNN}$ segmentation results. For instance, using the 5 best features (i.e. the 4 described ones plus entropy, ranked 5th) does not lead to better results compared to using only the first 4 features. This motivates the relevance of identifying the very best candidates in order to involve a limited set of features (which also makes sense wrt computational efficiency).

Finally, we observe that the RF ranking mainly follows the CNN ranking for the features used standalone. The only exception is the case of $M G B$, ranked first by RF whereas being less efficient than some of the texture features. A possible cause of this phenomenon could be the potential ability of a CNN to generate in its first layers

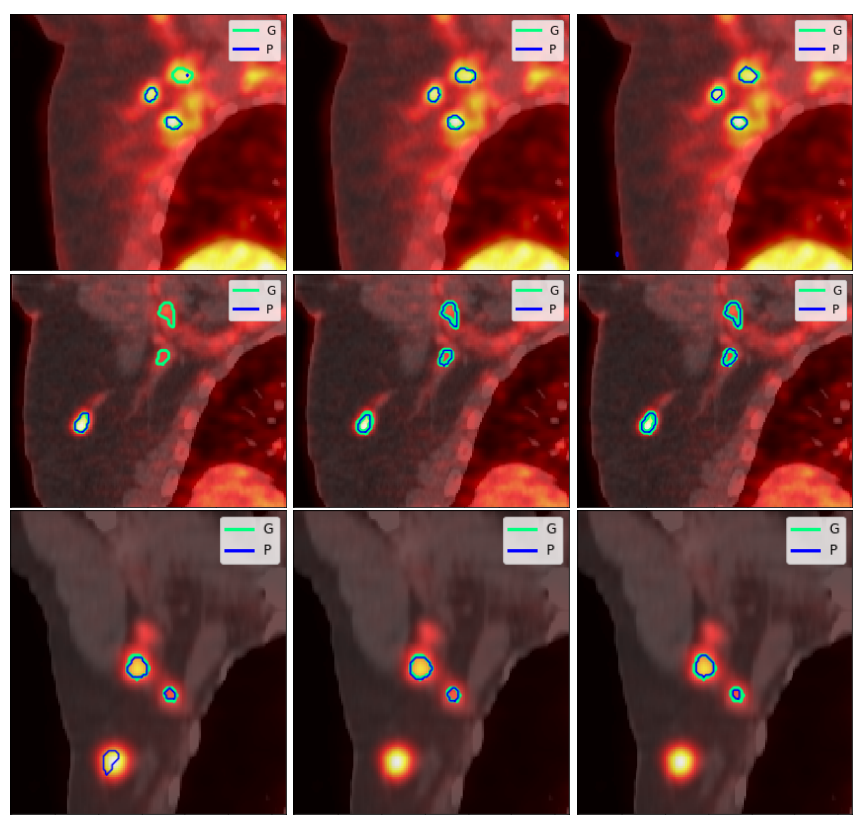

Fig. 3. Segmentation results (coronal slices of axillary regions, fused PET/CT image samples). From left to right: PET/CT (baseline); $\mathrm{PET} / \mathrm{CT}+$ Ine $; \mathrm{PET} / \mathrm{CT}+4$ features. Boundaries of predictions (blue) and ground-truth (green).

the information carried by this (merely linear) feature, compared to the more complex texture features, that may be infered only in much deeper layers.

\section{CONCLUSION}

We proposed a RF-based framework for selecting the best features that may be involved as input of U-Net architectures for segmentation of LN in PET/CT. This framework could, of course, be considered for other kinds of data and other $\mathrm{CNN}$ architectures.

Short-term perspectives will consist of carrying out more exhaustive experiments, with more data and other kinds of features (e.g. statistical atlases of pathological and anatomical structures).

We will also investigate the "relevance" of the tested features. Indeed, the purpose is to consider features that are likely to enrich a CNN. In other words, we will aim at using features that cannot be easily computed / approximated by CNNs in their first layers. This will lead us to develop a framework for assessing the ability of a $\mathrm{CNN}$ to infer different types of feature maps. 


\section{COMPLIANCE WITH ETHICAL STANDARDS}

This research study was conducted retrospectively using patient data made available in restricted access by Institut Jean-Godinot (Reims, France). All the patients had given a written consent for using their medical files for research purpose.

\section{ACKNOWLEDGMENTS}

This work was supported by the French Association Nationale Recherche Technologie (ANRT). The authors have no relevant financial or non-financial interests to disclose.

\section{REFERENCES}

[1] U. Nestle, S. Kremp, and A. Schaefer-Schuler et al., "Comparison of different methods for delineation of ${ }^{18}$ F-FDG PETpositive tissue for target volume definition in radiotherapy of patients with non-small cell lung cancer," Journal of Nuclear Medicine, vol. 46, pp. 1342-1348, 2005.

[2] T. Shepherd, M. Teräs, and R. Beichel et al., "Comparative study with new accuracy metrics for target volume contouring in PET image guided radiation therapy," IEEE Transactions on Medical Imaging, vol. 31, pp. 2006-2024, 2012.

[3] B. Foster, U. Bagci, and A. Mansoor et al., "A review on segmentation of positron emission tomography images," Computers in Biology and Medicine, vol. 50, pp. 76-96, 2014.

[4] J.L. Vercher-Conejero, L. Pelegrí-Martinez, and D. LopezAzna et al., "Positron emission tomography in breast cancer," Diagnostics, vol. 5, pp. 61-83, 2015.

[5] R. Piva, F. Ticconi, and V. Ceriani et al., "Comparative diagnostic accuracy of 18F-FDG PET/CT for breast cancer recurrence," Breast Cancer: Targets and Therapy, vol. 9, pp. 461, 2017.

[6] I. Nogues, L. Lu, and X. Wang et al., "Automatic lymph node cluster segmentation using holistically-nested neural networks and structured optimization in CT images," in MICCAI, 2016, pp. 388-397.

[7] A. Barbu, M. Suehling, and X. Xu et al., "Automatic detection and segmentation of axillary lymph nodes," in MICCAI, 2010, pp. 28-36.

[8] A. Krizhevsky, I. Sutskever, and G. E. Hinton, "ImageNet classification with deep convolutional neural networks," in NeurIPS, 2012, pp. 1106-1114.

[9] J. Long, E. Shelhamer, and T. Darrell, "Fully convolutional networks for semantic segmentation," in CVPR, 2015, pp. 3431-3440.

[10] G. J. S. Litjens, T. Kooi, and B. E. Bejnordi et al., "A survey on deep learning in medical image analysis," Medical Image Analysis, vol. 42, pp. 60-88, 2017.

[11] O. Ronneberger, P. Fischer, and T. Brox, "U-Net: Convolutional networks for biomedical image segmentation," in $M I C$ CAI, 2015, pp. 234-241.

[12] H. Wang, Z. Zhou, and Y. Li et al., "Comparison of machine learning methods for classifying mediastinal lymph node metastasis of non-small cell lung cancer from ${ }^{18}$ F-FDG PET/CT images," EJNMMI Research, vol. 7, pp. 11, 2017.
[13] Z. Zhong, Y. Kim, and L. Zhou et al., "3D fully convolutional networks for co-segmentation of tumors on PET-CT images," in $I S B I, 2018$, pp. 228-231.

[14] B. E. Bejnordi, M. Veta, and P. J. van Diest et al., "Diagnostic assessment of deep learning algorithms for detection of lymph node metastases in women with breast cancer," Journal of the American Medical Association, vol. 318, pp. 2199-2210, 2017.

[15] D. L. Farfan Cabrera, N. Gogin, and D. Morland et al., "Segmentation of axillary and supraclavicular tumoral lymph nodes in PET/CT: A hybrid CNN/component-tree approach," in ICPR, 2020, https://hal.archives-ouvertes.fr/hal-02963814.

[16] E. J. Breen and R. Jones, "Attribute openings, thinnings, and granulometries," Computer Vision and Image Understanding, vol. 64, pp. 377-389, 1996.

[17] P. Salembier, A. Oliveras, and L. Garrido, "Anti-extensive connected operators for image and sequence processing," IEEE Transactions on Image Processing, vol. 7, pp. 555-570, 1998.

[18] É. Grossiord, N. Passat, and H. Talbot et al., "Shaping for PET image analysis," Pattern Recognition Letters, vol. 131, pp. 307-313, 2020.

[19] É. Grossiord, H. Talbot, and N. Passat et al., "Hierarchies and shape-space for PET image segmentation," in ISBI, 2015, pp. 1118-1121.

[20] F. J. Alvarez Padilla, B. Romaniuk, and B. Naegel et al., "Hierarchical forest attributes for multimodal tumor segmentation on FDG-PET/contrast-enhanced CT," in ISBI, 2018, pp. 163167.

[21] F. J. Alvarez Padilla, B. Romaniuk, and B. Naegel et al., "Matching filtering by region-based attributes on hierachical structures for image co-segmentation," in ICIP, 2018, pp. 131135.

[22] H. Urien, I. Buvat, and N. Rougon et al., "Brain lesion detection in 3D PET images using max-trees and a new spatial context criterion," in ISMM, 2017, pp. 455-466.

[23] F. J. Alvarez Padilla, É. Grossiord, and B. Romaniuk et al., "Multicriteria 3D PET image segmentation," in IPTA, 2015, pp. 346-351.

[24] É. Grossiord, H. Talbot, and N. Passat et al., "Automated 3D lymphoma lesion segmentation from PET/CT characteristics," in $I S B I, 2017$, pp. 174-178.

[25] L. Breiman, "Random forests," Machine Learning, vol. 45, pp. 5-32, 2001.

[26] A. Dufour, O. Tankyevych, and B. Naegel et al., "Filtering and segmentation of 3D angiographic data: Advances based on mathematical morphology," Medical Image Analysis, vol. 17, pp. 147-164, 2013.

[27] R. Jones, "Connected filtering and segmentation using component trees," Computer Vision and Image Understanding, vol. 75, pp. 215-228, 1999.

[28] E. Carlinet and T. Géraud, "A comparative review of component tree computation algorithms," IEEE Transactions on Image Processing, vol. 23, pp. 3885-3895, 2014.

[29] R. M. Haralick, "Statistical and structural approaches to texture," Proceedings of the IEEE, vol. 67, pp. 786-804, 1979.

[30] N. V. Chawla, K. W. Bowyer, and L. O. Hall et al., "SMOTE: synthetic minority over-sampling technique," Journal of Artificial Intelligence Research, vol. 16, pp. 321-357, 2002. 